\title{
HYDROGEN FLUORIDE IN HIGH-MASS STAR-FORMING REGIONS
}

\author{
M. EMPrechtinger ${ }^{1}$, R. R. MONJE ${ }^{1}$, F. F. S. van DeR TAK ${ }^{2}$, M. H. D. vAN DER WieL ${ }^{2,3,4}$, D. C. Lis ${ }^{1}$, \\ D. Neufeld 5 , T. G. Phillips ${ }^{1}$, AND C. CeCCARelli ${ }^{6}$ \\ ${ }^{1}$ California Institute of Technology, Cahill Center for Astronomy and Astrophysics 301-17, Pasadena, CA 91125, USA; emprecht@ caltech.edu \\ ${ }^{2}$ SRON Netherlands Institute for Space Research, NL-9747 AD Groningen, The Netherlands \\ ${ }^{3}$ Institute for Space Imaging Science, Department of Physics and Astronomy, University of Lethbridge, Lethbridge, AB T1K 3M4, Canada \\ ${ }^{4}$ Kapteyn Astronomical Institute, University of Groningen, NL-9700 AV Groningen, The Netherlands \\ 5 Department of Physics and Astronomy, Johns Hopkins University, Baltimore, MD 21218, USA \\ ${ }^{6}$ UJF-Grenoble 1/CNRS-INSU, Institut de Planétologie et d'Astrophysique de Grenoble (IPAG) UMR 5274, F-38041 Grenoble, France \\ Received 2011 November 1; accepted 2012 June 24; published 2012 August 22
}

\begin{abstract}
Hydrogen fluoride (HF) has been established to be an excellent tracer of molecular hydrogen in diffuse clouds. In denser environments, however, the HF abundance has been shown to be approximately two orders of magnitude lower. We present Herschel/HIFI observations of HF $J=1-0$ toward two high-mass star formation sites, NGC 6334 I and AFGL 2591. In NGC 6334 I the HF line is seen in absorption in foreground clouds and the source itself, while in AFGL $2591 \mathrm{HF}$ is partially in emission. We find an $\mathrm{HF}$ abundance with respect to $\mathrm{H}_{2}$ of $1.5 \times 10^{-8}$ in the diffuse foreground clouds, whereas in the denser parts of NGC $6334 \mathrm{I}$ we derive a lower limit on the HF abundance of $5 \times 10^{-10}$. Lower HF abundances in dense clouds are most likely caused by freezeout of HF molecules onto dust grains in high-density gas. In AFGL 2591, the view of the hot core is obstructed by absorption in the massive outflow, in which HF is also very abundant $\left(3.6 \times 10^{-8}\right)$ due to the desorption by sputtering. These observations provide further evidence that the chemistry of interstellar fluorine is controlled by freezeout onto gas grains.
\end{abstract}

Key words: ISM: molecules - stars: formation

Online-only material: color figures

\section{INTRODUCTION}

Hydrogen fluoride (HF) is an exceptional molecule because of its peculiar chemistry. Fluorine is one of the few atoms which has a greater affinity to hydrogen than hydrogen itself, and thus the reaction

$$
\mathrm{H}_{2}+\mathrm{F} \rightarrow \mathrm{HF}+\mathrm{H}
$$

is highly exothermic $(\Delta E \approx 16,000 \mathrm{~K}$, although the activation of this reaction energy is $400 \mathrm{~K}$ and quantum mechanical tunneling through this barrier has to be considered). The destruction of HF occurs by the relatively slow photodissociation $\left(1.17 \times 10^{-10} \mathrm{~s}^{-1}\right)$ and reactions with ions, such as $\mathrm{He}^{+}, \mathrm{H}_{3}^{+}$, and $\mathrm{C}^{+}$, which are rare compared to $\mathrm{H}_{2}$. Thus, $\mathrm{HF}$ formation is much more efficient than its destruction under most interstellar conditions. As a consequence HF is considered to be the main reservoir of fluorine in molecular regions, i.e., where $\mathrm{H}_{2} / \mathrm{H}>1$, and therefore it is thought to be an ideal tracer of $\mathrm{H}_{2}$. Astrochemical models predict an abundance of HF relative to $\mathrm{H}_{2}$ (all fractional abundances given in this paper are relative to $\mathrm{H}_{2}$ ) of $3.6 \times 10^{-8}$ in such clouds (Neufeld et al. 2005).

The first detection of HF was reported by Neufeld et al. (1997), who observed the HF $J=2-1$ transition ( $v=$ $2463.43 \mathrm{GHz})$ with relatively low resolution $(R=9600)$ in absorption toward Sgr B2, using the Infrared Space Observatory. However, because high densities or strong radiation fields are needed to populate the HF $J=1$ level, the HF column density derived from this observation is subject to large uncertainties. With the advent of the HIFI instrument (de Graauw et al. 2010) aboard the Herschel Space Observatory (Pilbratt et al. 2010), high-resolution spectroscopy $\left(R>10^{6}\right)$ of the ground-state rotational transition of HF has become possible for the first time.
Several HF observations in diffuse cloud along the lines of sight toward strong submillimeter dust continuum sources, such as W31C (Neufeld et al. 2010), W49N and W51 (Sonnentrucker et al. 2010), and Sgr B2(M) (Monje et al. 2011) have been reported. HF $J=1-0$ is detected in absorption toward all of these continuum sources, which was expected considering the extreme conditions necessary to populate the excited rotational states of this molecule. These observations prove that HF is an excellent tracer of $\mathrm{H}_{2}$ in diffuse clouds, although the abundances of $\mathrm{HF}$ relative to $\mathrm{H}_{2}$, derived by comparison with $\mathrm{CH}$, are $\sim 1.5 \times 10^{-8}$, with an uncertainty of $46 \%$, and thus a factor of two below the chemical model predictions. Furthermore, $\mathrm{HF}$ turned out to be a much more sensitive tracer of $\mathrm{H}_{2}$ than the commonly used $\mathrm{CO}$, which is in agreement with the model prediction that $\mathrm{HF}$ is more abundant than $\mathrm{CO}$ in diffuse clouds with low extinction (Neufeld et al. 2005). Sonnentrucker et al. (2010), for example, detected a diffuse cloud on the line of sight toward W51, not seen in CO.

The only HF abundance calculated in a high-mass starforming region, and not in a diffuse foreground cloud, has been reported by Phillips et al. (2010), who detected HF $J=1-0$ in absorption toward Orion KL. Orion KL is a peculiar source, heated from the front, which shows hardly any lines in absorption at submillimeter wavelengths. The HF abundance derived by Phillips et al. is a lower limit of $1.6 \times 10^{-10}$, approximately two orders of magnitude lower than the HF abundances reported in diffuse clouds. This result can be either explained by geometrical effects, due to the incomplete coverage of the continuum source by absorbing material, or, under the assumption that most fluorine is bound in HF, is caused by HF molecules sticking onto the surfaces of dust grains under highdensity conditions. The only three detections of HF in emission 
so far are in the immediate vicinity of the AGB-star IRC+10216 (Agúndez et al. 2011), the Orion Bar (Van der Tak et al. 2012), and toward the Seyfert 1 Galaxy Mrk 231 (Van der Werf et al. 2010).

To investigate the abundances of $\mathrm{HF}$ in dense regions in more detail, we analyze here the HF $J=1-0$ spectra toward two high-mass star-forming regions, NGC 6334 I and AFGL 2591. NGC 6334 is a relatively nearby $(1.7 \mathrm{kpc}$; Neckel 1978) high-mass star-forming region, which harbors sites of many stages of protostellar evolution (Straw \& Hyland 1989). Singledish continuum observations at submillimeter wavelength show that a total mass of $200 M_{\odot}$ is associated with NGC 6334 I (Sandell 2000). The molecular hot core NGC 6334 I, studied extensively over the last decades (e.g., Beuther et al. 2008, 2007; Hunter et al. 2006), is associated with an ultracompact H II region (de Pree et al. 1995) and shows a very line-rich spectrum (Schilke et al. 2006, Thorwirth et al. 2003). HIFI observations in NGC 6334 I have led to the detection of CH (Van der Wiel et al. 2010), $\mathrm{H}_{2} \mathrm{O}$ (Emprechtinger et al. 2010), $\mathrm{H}_{2} \mathrm{O}^{+}$ (Ossenkopf et al. 2010), and $\mathrm{H}_{2} \mathrm{Cl}^{+}$(Lis et al. 2010), showing that hydrides are common toward this source. Furthermore, a bipolar outflow (Leurini et al. 2006; Beuther et al. 2008) and $\mathrm{H}_{2} \mathrm{O}, \mathrm{OH}, \mathrm{CH}_{3} \mathrm{OH}$ class II, and $\mathrm{NH}_{3}$ masers have been detected (e.g., Kraemer \& Jackson 1995; Ellingsen et al. 1996; Walsh et al. 2007). Rolffs et al. (2011) studied the radial structure of several high-mass star-forming cores, including NGC 6334 I. This investigation, mainly based on HCN observations with the APEX telescope, revealed a density law of $n \propto r^{-1.5}$. The temperature also follows a power law, with an index of 0.83 and 0.4 in the inner and the outer part, respectively. Interferometric data (Submillimeter Array; SMA), however, revealed that the internal structure of NGC $6334 \mathrm{I}$ is much more complex. The hot core consists of four compact condensations within a $10^{\prime \prime}$ region, emitting about $50 \%$ of the continuum flux, whereas the other $50 \%$ stems from the extended envelope (Hunter et al. 2006).

AFGL 2591 is an isolated high-mass star-forming region located within the Cygnus-X region. The distance toward AFGL 2591 was reported to be between $0.5 \mathrm{kpc}$ and $2.0 \mathrm{kpc}$, and for modeling purposes a distance of $1 \mathrm{kpc}$ was assumed (e.g., Van der Tak et al. 1999). However, new measurements based on very long baseline interferometry parallax measurements of $\mathrm{H}_{2}$ masers suggest that the distance might be as large as $3.3 \mathrm{kpc}$ (Rygl et al. 2012). Based on a distance of $1 \mathrm{kpc}$, the luminosity of AFGL 2591 is $\sim 2 \times 10^{4} L_{\odot}$; however, adopting the newly measured distances the luminosity would be approximately a factor 10 higher. The embedded central star, which is obscured in the visible by the massive envelope, has an estimated mass of $16 M_{\odot}$ and an effective temperature of 33,000 K (Van der Tak \& Menten 2005). Besides the circumstellar envelope, a massive outflow with an outflow velocity of $\sim 20 \mathrm{~km} \mathrm{~s}^{-1}$ with respect to the systemic velocity has been detected (Lada et al. 1984). In addition, Hasegawa \& Mitchell (1995) found a second, faster $\left(v_{\text {out }} \approx 40 \mathrm{~km} \mathrm{~s}^{-1}\right.$ ), but weaker outflow. Many attempts to model the structure of AFGL 2591 have been carried out assuming spherical symmetry and a power-law density and temperature structure (e.g., Van der Tak et al. 1999, 2000; Doty et al. 2002; de Wit et al. 2009). A cavity caused by the outflow might be present (Van der Tak et al. 1999). Van der Wiel et al. (2011) mapped AFGL 2591 in the frequency range from 330 to $373 \mathrm{GHz}$ with the James Clerk Maxwell telescope. In this frequency range they found $\sim 160$ lines, of which 35 show extended emission $\left(>15^{\prime \prime}\right)$. These observations reveal a smallscale structure $\left(\leqslant 10^{4} \mathrm{AU}\right)$, and a line-of-sight velocity gradient is apparent in most molecules. Their modeling suggests that a non-isotropic structure or a velocity gradient must be present on $\sim 10^{4}$ AU scales in order to explain the observations.

\section{OBSERVATIONS}

The observations used in this paper are part of the Chemical Herschel Surveys of Star-forming regions (Ceccarelli et al. 2010), a Herschel guaranteed time key program, which has conducted unbiased spectral line surveys of several star-forming regions. The HF $J=1-0$ spectra presented in this paper are observed in two sources: NGC 6334 I $\left(\alpha_{2000}: 17^{\mathrm{h}} 20^{\mathrm{m}} 53^{\mathrm{s}} .32, \beta_{2000}:-35^{\circ} 46^{\prime} 58^{\prime \prime} .5\right)$ and AFGL 2591 $\left(\alpha_{2000}: 20^{\mathrm{h}} 29^{\mathrm{m}} 24^{\mathrm{s}} .9, \beta_{2000}:+40^{\circ} 11^{\prime} 21^{\prime \prime}\right.$. 6$)$. The observation identification (ObsID) numbers of the scans are 1342206594 and 1342196510 for the observations in NGC 6334 I and AFGL 2591, respectively. Data were taken on 2010 May 12 (AFGL 2591) and 2010 October 14 (NGC 6334 I).

Both observations were carried out with the HIFI instrument (de Graauw et al. 2010) on board the Herschel Space Observatory (Pilbratt et al. 2010) using the double beam switch mode (180" chopper throw), and have been reduced with the HIPE pipeline (Ott 2010) version 5.1 and version 3.0, respectively. The double sideband (DSB) spectra were observed with a redundancy of eight, which allows the deconvolution and isolation of the single sideband (SSB) spectra (Comito $\&$ Schilke 2002). In the case of NGC 6334 I the deconvolution was done in HIPE and the deconvolved SSB spectra were exported to the FITS format for subsequent analyses in the IRAM/GILDAS package. ${ }^{7}$ The spectra of AFGL 2591 were exported to the FITS format right after the basic data reduction (level 2), and further data reduction and deconvolution were done using GILDAS. The spectra shown in this paper are equally weighted averages of the $\mathrm{H}$ and $\mathrm{V}$ polarization.

The HF $J=1-0$ line $(v=1232.47627 \mathrm{GHz})$ has been clearly detected in both sources. The beam size of Herschel at this frequency is $18^{\prime \prime}$ and a main beam efficiency of 0.62 was applied. Both spectra are displayed in Figure 1.

\section{RESULTS AND ANALYSIS}

As shown in Figure 1, the HF line seen is in absorption, in both sources, over a wide range of velocities. Only in the velocity interval from $-8 \mathrm{~km} \mathrm{~s}^{-1}$ to $-2 \mathrm{~km} \mathrm{~s}^{-1}$ in AFGL 2591 is a weak emission seen. In many cases the HF absorption is completely saturated. Assuming that all HF molecules are in the rotational ground state, one can calculate from the absorption depth the line opacity and subsequently the HF column density using the following formulas:

$$
\tau=-\ln \left(\frac{T_{\mathrm{MB}}}{T_{\text {back }}}\right),
$$

where $T_{\mathrm{MB}}$ is the measured brightness temperature and $T_{\text {back }}$ is the temperature of the background continuum, and

$$
N_{\mathrm{HF}}=\frac{8 \pi^{3 / 2} \cdot \Delta v}{2 \sqrt{\ln 2} \lambda^{3} \cdot A} \frac{g_{l}}{g_{u}} \cdot \tau,
$$

where $\lambda$ is the wavelength $(243.2 \mu \mathrm{m}), A$ the Einstein $A$-coefficient $\left(2.422 \times 10^{-2} \mathrm{~s}^{-1}\right)$, and $g_{l}$ and $g_{u}$ are the statistical weights of the lower and upper states ( 1 and 3 , respectively).

\footnotetext{
www.iram.fr/IRAMFR/GILDAS/
} 

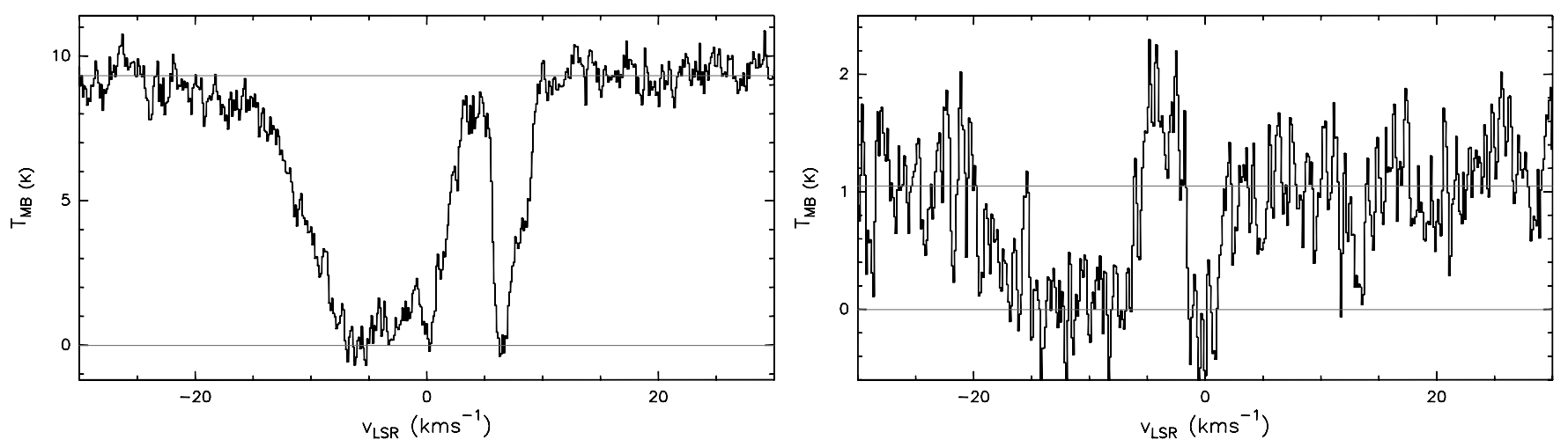

Figure 1. HF $J=1-0$ spectra in NGC 6334 I (left) and AFGL 2591 (right). The respective continuum levels as well as the zero levels are marked with the gray lines.

The assumption of all HF molecules being in the ground state is justified by the high Einstein $A$-coefficient and the low rates for collisional excitation (Guillon et al. 2012), corresponding to a critical density of $n_{\text {crit }}=1.9 \times 10^{8} \mathrm{~cm}^{-3}$, and the relatively high energy of the $J=1$ state $(50 \mathrm{~K})$. As shown by F. Van der Tak (submitted; arXiv:1203.6813), most alternative, non-thermal excitation mechanisms, such as infrared pumping and formation pumping, play only a negligible role. Excitation caused by submillimeter radiation, on the other hand, is possible in the hot inner part of high-mass star-forming regions and has to be considered. We will discuss the potential influence of this mechanism on the HF excitation individually for each source later on

\subsection{NGC 6334 I}

Many of the features seen in the HF spectrum toward NGC 6334 I originate from four foreground clouds, of which two reside in the immediate environment of NGC 6334 I, whereas the two remaining clouds are not associated with the source itself. These foreground clouds, which have been identified in CH spectra (Van der Wiel et al. 2010) are at velocities of $-3.0 \mathrm{~km} \mathrm{~s}^{-1}, 0.0 \mathrm{~km} \mathrm{~s}^{-1},+6.5 \mathrm{~km} \mathrm{~s}^{-1}$, and $+8.0 \mathrm{~km} \mathrm{~s}^{-1}$, whereas the velocities of the individual components of NGC 6334 I itself are between $-6.0 \mathrm{~km} \mathrm{~s}^{-1}$ and $-8.0 \mathrm{~km} \mathrm{~s}^{-1}$. From fitting several $\mathrm{C}^{18} \mathrm{O}$ lines observed with HIFI, we know that the source itself can be modeled by three components at $v_{\mathrm{LSR}}=-6.0 \mathrm{~km} \mathrm{~s}^{-1}$, $-8.0 \mathrm{~km} \mathrm{~s}^{-1}$, and $-6.5 \mathrm{~km} \mathrm{~s}^{-1}$ (R. Plume 2011, private communication). The first two components are identified as two of the embedded subcores (SMA 1 and SMA 2 following Hunter et al. 2006), whereas the latter component represents the lower density envelope. Because HF is a sensitive tracer of the molecular gas, we expect to see all these components in the HF spectrum as well. A massive outflow, detected in water (M. Emprechtinger et al., submitted) and CO (Leurini et al. 2006) in the velocity range between $-100 \mathrm{~km} \mathrm{~s}^{-1}$ and $+60 \mathrm{~km} \mathrm{~s}^{-1}$, is not seen in HF, neither in emission nor in absorption. The upper limit of the optical depth of HF in the blue lobe is 0.88 , which results in an upper limit on the HF column density of $8.42 \times 10^{13} \mathrm{~cm}^{-2}$, and therefore in an upper limit on the HF abundance of $5 \times 10^{-8}$, using the $\mathrm{H}_{2}$ column density given by Leurini et al. (2006). The upper limit of the integrated intensity of the red lobe is $2.69 \mathrm{~K} \mathrm{~km} \mathrm{~s}^{-1}$, which results, assuming the canonical HF abundance, in an upper limit on the HF excitation temperature of $10 \mathrm{~K}$. Both limits are consistent with standard HF abundances and negligible HF excitation. Thus, the non-detection of the outflow in HF is another indication that most HF molecules are
Table 1

Fit Results for the Individual Velocity Components in NGC 6334 I

\begin{tabular}{lccc}
\hline \hline Component & $\begin{array}{c}v v \\
\left(\mathrm{~km} \mathrm{~s}^{-1}\right)\end{array}$ & $\begin{array}{c}\Delta v \\
\left(\mathrm{~km} \mathrm{~s}^{-1}\right)\end{array}$ & $\begin{array}{c}N \\
\left(\mathrm{~cm}^{-2}\right)\end{array}$ \\
\hline Foreground & -3.0 & 5.0 & $2.4 \pm 1.2 \times 10^{13}$ \\
Foreground & 0.0 & 3.0 & $1.5 \pm 0.3 \times 10^{13}$ \\
Foreground & +6.5 & 1.2 & $>1.4 \times 10^{13}$ \\
Foreground & +8.0 & 2.5 & $4.0 \pm 0.8 \times 10^{12}$ \\
Envelope cloud & -6.5 & 4.0 & $2.7 \pm 1.3 \times 10^{13}$ \\
SMA 1 & -6.0 & 6.0 & $2.0 \pm 1.0 \times 10^{14}$ \\
SMA 2 & -8.0 & 5.0 & $2.0 \pm 1.0 \times 10^{14}$ \\
\hline$f_{c 1}=0.32 \pm 0.06$ & & $f_{c 2}=0.21 \pm 0.06$ &
\end{tabular}

in the ground state in this source. The foreground components and the envelope are expected to be more extended than the continuum sources in NGC $6334 \mathrm{I}$, and thus $T_{\text {back }}$ in Equation (1) is equal to the measured continuum level in the spectra. The components associated with embedded cores, however, absorb only continuum emitted from the respective core. Furthermore, we cannot be certain that the absorbing material covers all the continuum radiation emitted by the core. Therefore, even at very high optical depths only a fraction of the background radiation is absorbed. From interferometric observations at $\lambda=1.3 \mathrm{~mm}$ (Hunter et al. 2006), we estimate the contribution of each of the cores to be approximately $25 \%$ of the total continuum flux. This $25 \%$ value is only a rough estimate, since the actual value depends on the exact dust temperature and the coverage of the continuum source. Therefore, we use the actual continuum contribution of the cores as free fitting parameters $f_{c 1}$ and $f_{c 2}$. Using the centroid velocities and line widths of the seven components derived from the $\mathrm{CH}$ and $\mathrm{C}^{18} \mathrm{O}$ observations, and assuming that all $\mathrm{HF}$ molecules are in the ground state, the only free parameters in addition to $f_{c 1}$ and $f_{c 2}$ are the HF column densities of the individual components. This approach is valid for all components except the $+8.0 \mathrm{~km} \mathrm{~s}^{-1}$ foreground cloud, which is narrower in $\mathrm{HF}\left(\Delta v=2.5 \mathrm{~km} \mathrm{~s}^{-1}\right)$ than in $\mathrm{CH}$ $\left(\Delta v=3.5 \mathrm{~km} \mathrm{~s}^{-1}\right)$. Using Equations (1) and (2) with the free parameters $f_{c 1}, f_{c 2}$ and the HF column densities as a model to fit the observed spectra, we used the $\chi^{2}$ method to determine the column densities listed in Table 1; results are shown in Figure 2. The reduced $\chi^{2}$ for the best-fit result is 1.07 , but all results with a reduced $\chi^{2}$ lower than four have to be considered as possible solutions. Thus, the HF column densities can be determined only with an accuracy of $20 \%-50 \%$ for each individual component.

The HF column densities of the $0 \mathrm{~km} \mathrm{~s}^{-1}$ and the $+8.0 \mathrm{~km} \mathrm{~s}^{-1}$ foreground components are well determined $( \pm 20 \%)$, whereas the $-3.0 \mathrm{~km} \mathrm{~s}^{-1}$ component is less constrained $( \pm 50 \%)$ due 


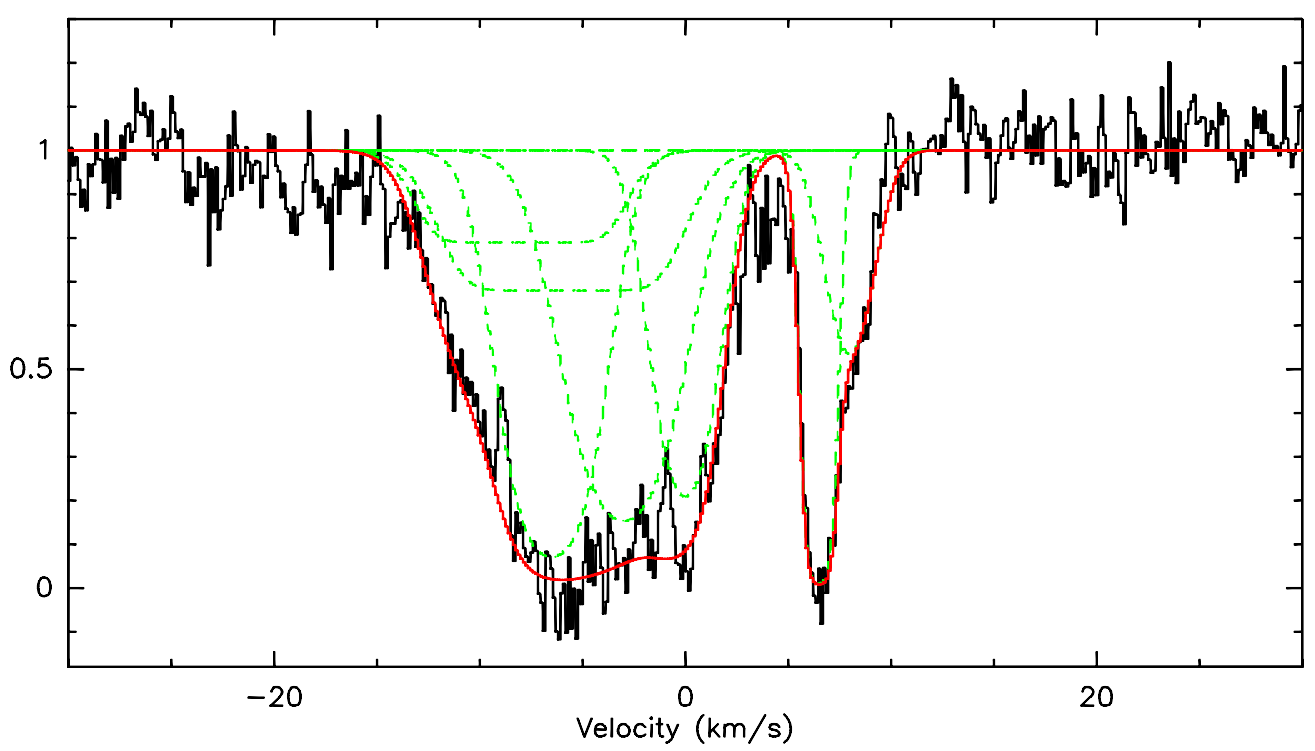

Figure 2. Comparison of the normalized spectrum of HF (black) and the model (red). The green lines show the individual components.

(A color version of this figure is available in the online journal.)

Table 2

Abundances of HF in the Foreground Components in NGC 6334 I

\begin{tabular}{rccr}
\hline \hline $\begin{array}{l}\text { Vel. Comp. } \\
\left(\mathrm{km} \mathrm{s}^{-1}\right)\end{array}$ & $\begin{array}{c}N(\mathrm{HF}) \\
\left(\mathrm{cm}^{-2}\right)\end{array}$ & $\begin{array}{c}N\left(\mathrm{H}_{2}\right) \\
\left(\mathrm{cm}^{-2}\right)\end{array}$ & \multicolumn{1}{c}{$X(\mathrm{HF})$} \\
\hline-3.0 & $2.40 \times 10^{13}$ & $1.8 \times 10^{21}$ & $1.3 \times 10^{-8}$ \\
0.0 & $1.50 \times 10^{13}$ & $6.0 \times 10^{20}$ & $2.5 \times 10^{-8}$ \\
+6.5 & $>1.4 \times 10^{13}$ & $8.6 \times 10^{20}$ & $>1.6 \times 10^{-8}$ \\
+8.0 & $4.0 \times 10^{12}$ & $6.0 \times 10^{20}$ & $6.7 \times 10^{-9}$ \\
\hline
\end{tabular}

to the blending with the absorption features from NGC 6334 I itself. For the $+6.5 \mathrm{~km} \mathrm{~s}^{-1}$ component only a lower limit for the column density can be given since this line is optically thick. However, the opacity of the $+6.5 \mathrm{~km} \mathrm{~s}^{-1}$ component is moderate because we do not see any effects of extremely high optical depth, such as a line width wider than the optically thin $\mathrm{CH}$ line, or a saturated line shape. Two narrow residuals seen at $v_{\mathrm{LSR}}=-1 \mathrm{~km} \mathrm{~s}^{-1}$ and $-9 \mathrm{~km} \mathrm{~s}^{-1}$ are not reproduced by our fit. Considering that both residuals are located in the wings of major absorption features, a possible explanation is a velocity or line width gradient within individual components. These two features may also be caused by emission lines from other species. However, there are no plausible candidates in the JPL database ${ }^{8}$ or the CDMS catalog (Müller et al. 2005). $\mathrm{CH}$ is known to be an excellent tracer of $\mathrm{H}_{2}$ at densities below $10^{4} \mathrm{~cm}^{-3}$ with an abundance of $3.5 \times 10^{-8}$ with respect to $\mathrm{H}_{2}$ (Sheffer et al. 2008). Using the $\mathrm{CH}$ column densities reported by Van der Wiel et al. (2010) to derive the $\mathrm{H}_{2}$ column density, we are able to determine the HF abundance relative to $\mathrm{H}_{2}$ in the diffuse foreground clouds (see Table 2).

The HF abundance in the clouds at $-3.0 \mathrm{~km} \mathrm{~s}^{-1}, 0.0 \mathrm{~km} \mathrm{~s}^{-1}$, and $+6.5 \mathrm{~km} \mathrm{~s}^{-1}$ agrees fairly well with the abundances found in other diffuse clouds (Monje et al. 2011, Sonnentrucker et al. 2010). However, the $+8.0 \mathrm{~km} \mathrm{~s}^{-1}$ component exhibits an $\mathrm{HF}$ abundance which is significantly lower. The $+8.0 \mathrm{~km} \mathrm{~s}^{-1}$ component is also the component in which the HF line is much narrower than $\mathrm{CH}$. Furthermore, the abundance of $\mathrm{CH}^{+}$ is significantly enhanced in this cloud compared to the other

\footnotetext{
8 http://spec.jpl.nasa.gov/home.html
}

foreground components (D. Lis et al., in preparation), indicating that the chemical and physical properties of the gas may be very different (Godard et al. 2009).

Since observations of Orion KL suggest that the abundance of $\mathrm{HF}$ in dense gas in high-mass star-forming regions is lower than in diffuse clouds (Phillips et al. 2010), we attempted to derive the $\mathrm{HF}$ abundance in the components associated with NGC 6334 I itself. The fits for the SMA 2 core and the envelope material are relatively good and HF column densities can be determined with an accuracy of 50\%. The column density of the SMA 1 core, however, is poorly constrained since this component is blended with several other components. As can be seen in Figure 2, the HF absorption features originating from SMA 1 and SMA 2 are largely overlapping, which results in a large ambiguity of the individual continuum coverage factors $( \pm 0.2)$. However, their sum can be defined quite well as $0.53 \pm 0.05$. The fit results for SMA 1 presented in Table 1 are based on the assumption that the $\mathrm{HF}$ abundance relative to $\mathrm{H}_{2}$ in SMA 1 and SMA 2, which have similar physical conditions, is the same. To estimate the $\mathrm{H}_{2}$ column density, we are using the $\mathrm{C}^{18} \mathrm{O}$ column densities derived by R. Plume (2011, private communication) from an LTE fit to seven $\mathrm{C}^{18} \mathrm{O}$ transitions. We are assuming standard values for the $\mathrm{C}^{16} \mathrm{O} / \mathrm{C}^{18} \mathrm{O}$ ratio $(500)$ and the $\mathrm{C}^{16} \mathrm{O}$ abundance $\left(9.5 \times 10^{-5}\right)$. Furthermore, we divide the resulting column density by two, because approximately half of the material emitting the $\mathrm{CO}$ lines resides behind the continuum sources, and therefore does not contribute to the HF absorption. The resulting HF abundances range from $3.7 \times 10^{-10}$ in the envelope to $(5-8) \times 10^{-10}$ in the dense cores. These values are approximately two orders of magnitude lower than the abundances in diffuse clouds and predicted by models (Neufeld et al. 2005) but comparable to the lower limits found by Phillips et al. (2010) in Orion KL. In these calculations we did not consider submillimeter radiative excitation, and therefore these results are lower limits. However, taking the observed continuum values (Hunter et al. 2006) and using large velocity gradient calculations, we found that we underestimate the HF column density by less than a factor four for conditions present in the dense cores. Therefore, the low abundances of HF derived in NGC 6334 I itself cannot be explained by $\mathrm{HF}$ excitation alone. 
The calculated HF abundances are based on the assumption that approximately half of the mass associated with NGC 6334 I resides in front of the corresponding continuum source. However, the actual structure of the source is not known, and thus the HF abundance might be underestimated. But it should be noted that it is very unlikely that the unknown geometry of NGC $6334 \mathrm{I}$ is the sole reason for the low HF abundance derived here. Assuming that only geometrical effects account for the low $\mathrm{HF}$ abundance would imply that, in all three components (SMA 1, SMA 2, and the envelope cloud) as well as in Orion $\mathrm{KL}$, the bulk of the molecular gas $(\sim 98 \%)$ resides behind the main continuum source.

\subsection{AFGL 2591}

The spectrum of AFGL 2591 is much simpler than that of NGC $6334 \mathrm{I}$; in addition to one foreground absorption component at $\sim 0 \mathrm{~km} \mathrm{~s}^{-1}$, only one broad component ranging from $-6 \mathrm{~km} \mathrm{~s}^{-1}$ to $-20 \mathrm{~km} \mathrm{~s}^{-1}$ can be seen. In the velocity range between the two absorption features a weak HF emission is detected. Both absorption features are most likely very optically thick, since all background radiation is completely absorbed in the corresponding velocity ranges. Unfortunately, the continuum of AFGL 2591 is much weaker than the one of NGC 6334 I and thus the signal-to-noise ratio is much lower, resulting in a lower limit for $\tau$ of 1.6.

The profile of the HF $J=1-0$ line is very peculiar and only the profiles of the water ground-state transitions $\mathrm{p}-\mathrm{H}_{2} \mathrm{O}$ $1_{11}-0_{00}$ and $\mathrm{p}-\mathrm{H}_{2} \mathrm{O} 2_{12}-1_{01}$ look somewhat similar, although the emission component is much stronger in the water lines (Choi et al. 2011). The broad absorption component is clearly blueshifted with respect to the systemic velocity of $-5.7 \mathrm{~km} \mathrm{~s}^{-1}$ of AFGL 2591, and thus most likely caused by the massive outflow. This outflow is also seen in $\mathrm{CO}$ and $\mathrm{HCO}^{+}$(Lada et al. 1984; Hasegawa \& Mitchell 1995), and centers at a velocity of $-7 \mathrm{~km} \mathrm{~s}^{-1}$, slightly blueshifted with respect to the envelope (Van der Wiel 2011). He also derived an $\mathrm{H}_{2}$ column density of the outflow of $1.5 \times 10^{22} \mathrm{~cm}^{-2}$, based on observations of several $\mathrm{CO}$ line and radiative transfer calculations. This $\mathrm{H}_{2}$ column density leads to an HF column density of $2 \times 10^{14} \mathrm{~cm}^{-2}$ assuming an HF abundance of $1.5 \times 10^{-8}$. Using a line width of $10 \mathrm{~km} \mathrm{~s}^{-1}$, which is the approximate velocity width of the outflow, this HF column density corresponds to a $\tau$ of $\sim 9$ (using Equation (2)), which is in agreement with the high optical depth seen in the observed spectrum. HF abundances of the order of $\sim 3 \times 10^{-10}$, as found in star-forming regions, are clearly too low to cause the observed deep absorption. This is a very interesting aspect, because the temperature $(100-150 \mathrm{~K})$ and density $\left(10^{5}-10^{6} \mathrm{~cm}^{-3}\right)$ are comparable to the conditions in high-mass star-forming regions. It should be noted that a shock with a velocity of $\approx 15 \mathrm{~km} \mathrm{~s}^{-1}$, as derived from the spectrum, may cause a partial dissociation of $\mathrm{H}_{2}$ (up to $80 \%$ ) due to energetic collisions with neutral particles (Flower \& Pineau Des Forêts 2010). This may cause an underestimation of the total hydrogen column density in the outflow $\left(2 \mathrm{~N}\left(\mathrm{H}_{2}\right)+\mathrm{N}(\mathrm{H})\right)$. However, collisions with enough energy to destroy $\mathrm{H}_{2}$ can potentially destroy $\mathrm{HF}$ and $\mathrm{CO}$ too. Furthermore, in the absence of $\mathrm{H}_{2}$, the HF production is hindered. Therefore, we are able to derive the $\mathrm{HF}$ abundance relative to $\mathrm{H}_{2}$, but not relative to the total hydrogen density. The error of the HF abundance relative to $\mathrm{H}_{2}$ is determined by the uncertainty of the $\mathrm{H}_{2}$ column density derived from $\mathrm{CO}$. We estimate that these errors are definitely less than the degree of the dissociation of $\mathrm{H}_{2}$ and therefore clearly lower than one order of magnitude.
The foreground absorption line width (equivalent width is $3.2 \mathrm{~km} \mathrm{~s}^{-1}$ ) is clearly larger than the corresponding feature in the CO lines ( $\Delta v=0.8 \mathrm{~km} \mathrm{~s}^{-1}$; Van der Wiel 2011), and more comparable to the width of the feature seen in $\mathrm{CH}\left(\Delta v=1.8 \mathrm{~km} \mathrm{~s}^{-1}\right.$; Bruderer et al. 2010). Using the line width of $\mathrm{CH}$ as the $\Delta v$ caused by Doppler broadening for HF, and fitting the absorption feature using Equations (1) and (2), leads to a minimum optical depth of 8 , and thus a minimum HF column density of $4 \times 10^{13} \mathrm{~cm}^{-2}$ in the foreground cloud. Comparing this HF column density with the $\mathrm{H}_{2}$ column density found by Van der Wiel (2011) based on CO observations yields an HF abundance relative to $\mathrm{H}_{2}$ of $>1.3 \times 10^{-8}$, which is a typical value found in diffuse clouds and close to that found in the clouds toward NGC 6334 I. However, the CO abundance in diffuse clouds might be significantly lower than the canonical value of $10^{-4}$. Sonnentrucker et al. (2007) found CO abundances with respect to $\mathrm{H}_{2}$ ranging from $2.75 \times 10^{-7}$ to $2.75 \times 10^{-5}$ in material on the line of sight toward several stars. Hence the total $\mathrm{H}_{2}$ column density toward AFGL 2591 may be underestimated and, because we derive only lower limits on the HF column density, the uncertainty of the derived HF abundance is very large.

To get a better understanding of the HF spectrum in AFGL 2591, especially of the outflow and the HF emission, we model this source using RATRAN (Hogerheijde \& Van der Tak 2000). RATRAN is a Monte Carlo radiative transfer code, which includes excitation by collision of the molecules with $\mathrm{H}_{2}$ and excitation by submillimeter radiation. It calculates a grid of spectra integrated along a pencil beam, which are subsequently convolved to the desired spatial resolution. The Einstein coefficients of the modeled transitions are taken from the LAMDA database (Schöier et al. 2005), whereas newly calculated collision rates are used (Guillon et al. 2012). The source model is based on the "static model" of Van der Wiel et al. (2011), who modeled the envelope of AFGL 2591 assuming a power-law density distribution $\left(n=r^{-1.0}\right)$ and used a selfconsistent solution for the dust temperature (Van der Tak et al. 2000). To take the outflow component into account, we add an expansion velocity to each shell in the model of Van der Wiel et al. (2011), which increases with radius as

$$
v_{\exp }=v_{\infty} \cdot\left(1-\frac{r_{0}}{r}\right)^{\beta},
$$

where $v_{\infty}$ is the maximum velocity of the outflow and $r_{0}$ is the radius at which the outflow starts. $\beta$, which was set to 2 , is a measure of how quickly the maximum velocity is reached; $r_{0}$ was set to a value so that the $\mathrm{H}_{2}$ column density outside this radius is comparable to $1.5 \times 10^{22} \mathrm{~cm}^{-2}$, the $N_{\mathrm{H}_{2}}$ found in the outflow (Van der Wiel 2011). At radii smaller than $r_{0}$ the expansion velocity is zero. The maximum velocity is set to $9 \mathrm{~km} \mathrm{~s}^{-1}$ to match the width of the absorption feature. The modeled HF spectrum is not very sensitive to the actual velocity profile, and a proper solution can be found for profiles with moderately increasing $v_{\text {exp }}$. The foreground component is fitted using the foreground option in RATRAN assuming an optical depth of 8 and a line width of $1.8 \mathrm{~km} \mathrm{~s}^{-1}$.

Deriving the HF abundance is somewhat difficult because the absorption features are optically thick. Hence, we calculate models with three different abundances relative to $\mathrm{H}_{2}$. In Model 1, we assume an abundance of $1.5 \times 10^{-8}$, the HF abundance found in diffuse clouds, whereas in Model 2 an HF abundance of $5 \times 10^{-10}$ as found in the dense part of AFGL 2591 is adopted. In Model 3, we assume a value of 


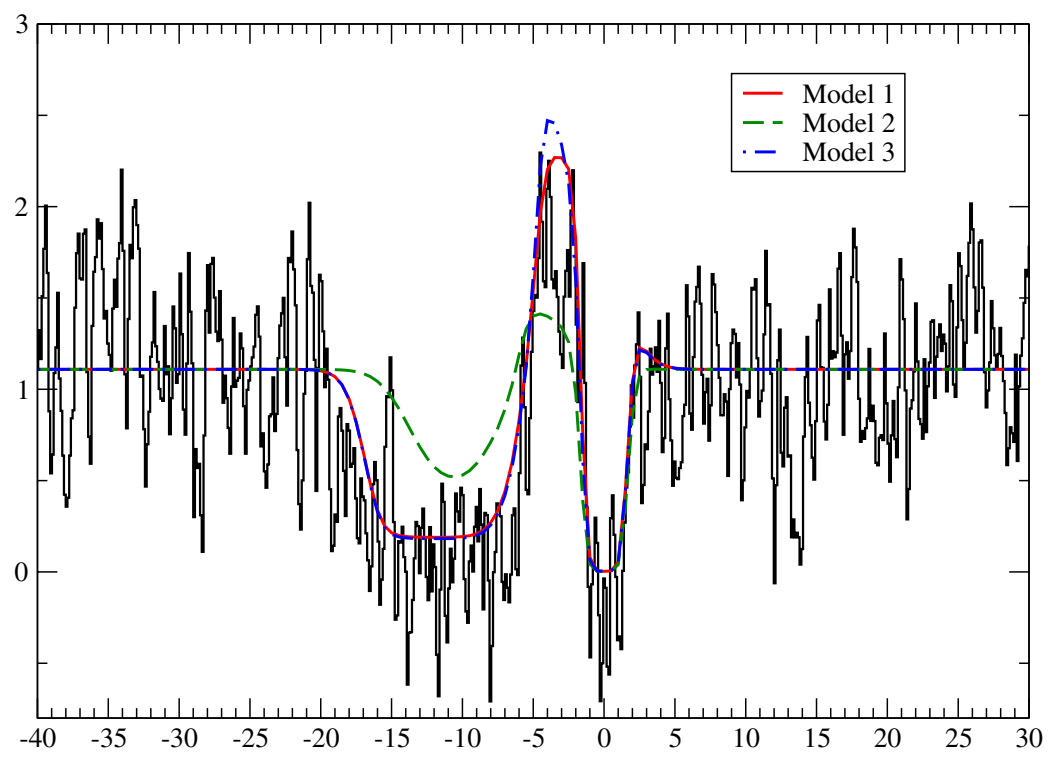

Figure 3. Comparison of the observed HF $J=1-0$ transition (black) with radiative transfer models. Model 1 (red): HF abundance is $1.5 \times 10^{-8}$; Model 2 (green): $\mathrm{HF}$ abundance is $5 \times 10^{-10}$; Model 3 (blue): $\mathrm{HF}$ abundance is $5 \times 10^{-10}$ in the quiescent gas and $3.6 \times 10^{-8}$ in the outflow.

(A color version of this figure is available in the online journal.)

$5 \times 10^{-10}$ in the inner part of the source, where $v_{\exp }=$ $0 \mathrm{~km} \mathrm{~s}^{-1}$, and $1.5 \times 10^{-8}$ in the outer part. A comparison of the three models with observations is shown in Figure 3. Models 1 and 3 fit the observations quite well, whereas an HF abundance as low as $5 \times 10^{-10}$ in the outflow can be ruled out. The model calculations also show that the emission seen at $v_{\mathrm{LSR}} \approx-4 \mathrm{~km} \mathrm{~s}^{-1}$ stems from the red lobe on the far side of the continuum source, and not from the hot core itself. The emission at $v_{\mathrm{LSR}} \approx-4 \mathrm{~km} \mathrm{~s}^{-1}$ is overestimated by both Model 1 and Model 3. This may be due to geometry effects, since the outflow is modeled by a shell, whereas it is in fact a collimated outflow. The absorption of all radiation in the blue lobe of the outflow indicates that most HF molecules are in the $J=0$ state, and thus excitation by collisions with $\mathrm{H}_{2}$ or any other effective excitation mechanism can be ruled out. As suggested by our model calculation, the emission from the red lobe is most likely caused by absorption and immediate reemission of $1.2 \mathrm{THz}$ photons from the continuum source of AFGL 2591.

Because none of the features of the HF spectra stems from the hot quiescent part of AFGL 2591, we cannot distinguish whether the abundance in the inner part is similar to the diffuse cloud abundance or to the lower limits found in the high-mass star-forming regions NGC 6334 I and Orion KL (Phillips et al. 2010). The assumption of a uniform, low HF abundance in the dense parts of high-mass star-forming regions is consistent with our data.

\section{SUMMARY AND DISCUSSION}

We determine the abundance of HF in very different physical environments, and obtain very different results. In agreement with previous studies, we find the $\mathrm{HF}$ abundance of $\sim 1.5 \times 10^{-8}$ in diffuse clouds, implying that about half of the available fluorine is in the form of HF in the gas phase. An exception is the $+8.0 \mathrm{~km} \mathrm{~s}^{-1}$ component toward NGC $6334 \mathrm{I}$, in which HF is less than half as abundant as in other diffuse clouds. The chemical composition of this foreground component, however, seems to be different from the other components (e.g., strongly enhanced $\mathrm{CH}^{+}$abundance; D. Lis et al., in preparation), which requires further studies.

In the high-mass star-forming regions, at much higher densities compared with diffuse clouds, the HF abundance seems to drop by about two orders of magnitude. The correlation with the physical conditions, especially density and temperature, would be crucial to determine the nature of this HF depletion, but it still remains to be found. Phillips et al. (2010) argue that the low abundance of HF at higher densities is due to freezeout of HF onto dust grains. This argument is drawn from the fact that almost all fluorine should be bound in HF due to its high proton affinity, and that the desorption energy of HF is assumed to be quite large due to its polar nature. In dense $\left(10^{5}-10^{6} \mathrm{~cm}^{-3}\right)$ and warm (100-150 K), but dynamically active, regions such as the outflow in AFGL 2591, we find HF abundances close to the values found in diffuse clouds. In the picture of HF freezeout at high densities, this can be explained by HF desorption due to sputtering by high-velocity particles, similar to the desorption of water in outflows (Kristensen et al. 2010; Emprechtinger et al. 2010).

In both sources HF is the only fluorine-bearing species we find in the HIFI line survey, and we can confirm non-detections for $\mathrm{CF}, \mathrm{CF}^{+}, \mathrm{DF}$, and $\mathrm{H}_{2} \mathrm{~F}^{+}$. In NGC $6334 \mathrm{I}$ the upper limit for $\mathrm{CF}^{+} J=5-4$, a molecule which has been detected in the Orion Bar with an abundance of a few times $10^{-10}$ (Neufeld et al. 2006), is $0.012 \mathrm{~K}$. Assuming cloud properties, as determined by $\mathrm{C}^{18} \mathrm{O}$ fits (R. Plume 2011, private communication), yields an upper limit for the $\mathrm{CF}^{+}$abundance of $6 \times 10^{-12}$. This low upper limit, 50 times lower than the abundance found in the Orion Bar, can be explained by the different nature of the two objects. $\mathrm{C}^{+}$, a precursor of $\mathrm{CF}^{+}$, and thus $\mathrm{CF}^{+}$itself are expected to be much more abundant in photodominated regions, such as the Orion Bar, than in massive cores. The upper limit for the abundance of these four fluorine-bearing species together is $\sim 7 \times 10^{-11}$, and thus these molecules hold less than $0.4 \%$ of the total fluorine. The non-detection of other fluorine-bearing species is another indication that freezeout onto dust grains causes the low HF abundances observed in dense, quiescent gas. 
This analysis demonstrates that the utility of HF as a tracer of $\mathrm{H}_{2}$, as predicted by chemical models, is only applicable to diffuse clouds. The fluorine chemistry in dense clouds is not yet fully understood and further theoretical and observational studies are required.

HIFI has been designed and built by a consortium of institutes and university departments from across Europe, Canada, and the United States under the leadership of SRON Netherlands Institute for Space Research, Groningen, The Netherlands, and with major contributions from Germany, France, and the USA. Consortium members are: Canada: CSA, University of Waterloo; France: CESR, LAB, LERMA, IRAM; Germany: KOSMA, MPIfR, MPS; Ireland: NUI Maynooth; Italy: ASI, IFSI-INAF, Osservatorio Astrofisico di Arcetri-INAF; The Netherlands: SRON, TUD; Poland: CAMK, CBK; Spain: Observatorio Astronómico Nacional (IGN), Centro de Astrobiología (CSICINTA); Sweden: Chalmers University of Technology-MC2, RSS \& GARD, Onsala Space Observatory, Swedish National Space Board, Stockholm University-Stockholm Observatory; Switzerland: ETH Zurich, FHNW; USA: Caltech, JPL, NHSC. We thank many funding agencies for financial support. Support for this work was provided by NASA through an award issued by JPL/Caltech, by l'Agence Nationale pour la Recherche (ANR), France (project FORCOMS, contracts ANR-08-BLAN022), and the Centre National d'Etudes Spatiales (CNES).

\section{REFERENCES}

Agúndez, M., Cernicharo, J., Waters, L. B. F. M., et al. 2011, A\&A, 533, L6 Beuther, H., Walsh, A. J., Thorwirth, S., et al. 2007, A\&A, 466, 989 Beuther, H., Walsh, A. J., Thorwirth, S., et al. 2008, A\&A, 481, 169 Bruderer, S., Benz, A. O., Van Dishoeck, E. F., et al. 2010, A\&A, 521, L44 Ceccarelli, C., Bacmann, A., Boogert, A., et al. 2010, A\&A, 521, L22 Choi, Y., Van der Tak, F., \& Van Dishoeck, E. F. 2011, in IAU Symp. 280, The Molecular Universe (Cambridge: Cambridge Univ. Press), 128P Comito, C., \& Schilke, P. 2002, A\&A, 395, 357

de Graauw, T., Helmich, F. P., Phillips, T. G., et al. 2010, A\&A, 518, L6 de Pree, C. G., Rodriguez, L. F., Dickel, H. R., \& Goss, W. M. 1995, ApJ, 447, 220

de Wit, W. J., Hoare, M. G., Fujiyoshi, T., et al. 2009, A\&A, 494, 157

Doty, S. D., Van Dishoeck, E. F., Van der Tak, F. F. S., \& Boonman, A. M. S. 2002, A\&A, 389, 446

Ellingsen, S. P., von Bibra, M. L., McCulloch, P. M., et al. 1996, MNRAS, 280, 378

Emprechtinger, M., Lis, D. C., Bell, T., et al. 2010, A\&A, 521, L28

Flower, D. R., \& Pineau Des Forêts, G. 2010, MNRAS, 406, 1745

Godard, B., Falgarone, E., \& Pineau Des Forêts, G. 2009, A\&A, 495, 847
Guillon, G., Benz, A. O., Van Dishoeck, E. F., et al. 2012, MNRAS, 420, 597 Hasegawa, T. I., \& Mitchell, G. F. 1995, ApJ, 451, 225

Hogerheijde, M. R., \& Van der Tak, F. F. S. 2000, A\&A, 362, 697

Hunter, T. R., Brogan, C. L., Megeath, S. T., et al. 2006, ApJ, 649, 888

Kraemer, K. E., \& Jackson, J. M. 1995, ApJ, 439, L9

Kristensen, L. E., Visser, R., Van Dishoeck, E. F., et al. 2010, A\&A, 521, L30

Lada, C. J., Thronson, H. A., Jr., Smith, H. A., Schwartz, P. R., \& Glaccum, W. 1984, ApJ, 286, 302

Leurini, S., Schilke, P., Parise, B., et al. 2006, A\&A, 454, L83

Lis, D. C., Pearson, J. C., Neufeld, D. A., et al. 2010, A\&A, 521, L9

Monje, R. R., Emprechtinger, M., Phillips, T. G., et al. 2011, ApJ, 734, L23

Müller, H. S. P., Schlöder, F., Stutzki, J., \& Winnewisser, G. 2005, J. Mol. Struct., 742, 215

Neckel, T. 1978, A\&A, 69, 51

Neufeld, D. A., Schilke, P., Menten, K. M., et al. 2006, A\&A, 454, L37

Neufeld, D. A., Sonnentrucker, P., Phillips, T. G., et al. 2010, A\&A, 518, L108

Neufeld, D. A., Wolfire, M. G., \& Schilke, P. 2005, ApJ, 628, 260

Neufeld, D. A., Zmuidzinas, J., Schilke, P., \& Phillips, T. G. 1997, ApJ, 488, L141

Ossenkopf, V., Müller, H. S. P., Lis, D. C., et al. 2010, A\&A, 518, L111

Ott, S. 2010, in ASP Conf. Ser. 434, Astronomical Data Analysis Software and Systems XIX, ed. Y. Mizumoto, K.-I. Morita, \& M. Ohishi (San Francisco, CA: ASP), 139

Phillips, T. G., Bergin, E. A., Lis, D. C., et al. 2010, A\&A, 518, L109

Pilbratt, G. L., Riedinger, J. R., Passvogel, T., et al. 2010, A\&A, 518, L1

Rolffs, R., Schilke, P., Wyrowski, F., et al. 2011, A\&A, 527, A68

Rygl, K. L. J., Brunthaler, A., Sanna, A., et al. 2012, A\&A, 539, A79

Sandell, G. 2000, A\&A, 358, 242

Schilke, P., Comito, C., Thorwirth, S., et al. 2006, A\&A, 454, L41

Schöier, F. L., Van der Tak, F. F. S., Van Dishoeck, E. F., \& Black, J. H. 2005, A\&A, 432, 369

Sheffer, Y., Rogers, M., Federman, S. R., et al. 2008, ApJ, 687, 1075

Sonnentrucker, P., Neufeld, D. A., Phillips, T. G., et al. 2010, A\&A, 521, L12

Sonnentrucker, P., Welty, D. E., Thorburn, J. A., \& York, D. G. 2007, ApJS, 168,58

Straw, S. M., \& Hyland, A. R. 1989, ApJ, 340, 318

Thorwirth, S., Winnewisser, G., Megeath, S. T., \& Tieftrunk, A. R. 2003, in ASP Conf. Ser. 287, Galactic Star Formation Across the Stellar Mass Spectrum, ed. J. M. De Buizer \& N. S. Van der Bliek (San Francisco, CA: ASP), 257

Van der Tak, F. F. S., \& Menten, K. M. 2005, A\&A, 437, 947

Van der Tak, F. F. S., Ossenkopf, V., Nagy, Z., et al. 2012, A\&A, 537, L10

Van der Tak, F. F. S., Van Dishoeck, E. F., Evans, N. J., II, Bakker, E. J., \& Blake, G. A. 1999, ApJ, 522, 991

Van der Tak, F. F. S., Van Dishoeck, E. F., Evans, N. J., II, \& Blake, G. A. 2000, ApJ, 537, 283

Van der Werf, P. P., Isaak, K. G., Meijerink, R., et al. 2010, A\&A, 518, L42

Van der Wiel, M. H. D. 2011, PhD thesis, Univ. Groningen

Van der Wiel, M. H. D., Van der Tak, F. F. S., Lis, D. C., et al. 2010, A\&A, 521, L43

Van der Wiel, M. H. D., Van der Tak, F. F. S., Spaans, M., et al. 2011, A\&A, 532, A88

Walsh, A. J., Longmore, S. N., Thorwirth, S., Urquhart, J. S., \& Purcell, C. R. 2007, MNRAS, 382, L35 